\title{
CARACTERIZAÇÃO DE UM TUBO DE IMPEDÂNCIA ACÚSTICA VIA COMPUTAÇÃO NATURAL
}

\section{CHARACTERIZATION OF AN ACOUSTIC IMPEDANCE TUBE USING NATURAL COMPUTER}

\author{
Igor Feliciani Merizio ${ }^{1}$; Fábio Roberto Chavarette ${ }^{1}$; Roberto Outa ${ }^{2}$ \\ ${ }^{1}$ Universidade Estadual Paulista - UNESP. ${ }^{2}$ Faculdade de tecnologia de Araçatuba- \\ FATEC \\ E-mail: $\quad$ igorfeliciani@gmail.com ; fabio.chavarette@unesp.br ; \\ roberto.outa@gmail.com.
}

RESUMO - O sistema de monitoramento saúde estrutural (SHM), um sistema que avalia o estado de estruturas aeronáuticas, civil ou mecânicas e dá a previsão de sua vida remanescente, surgiu com a necessidade de dar mais viabilidade econômica ao monitoramento de estruturas, e na prevenção de falhas. Assim, define-se tal sistema como uma medida profilática, confiável e eficaz, contra a falha estrutural. Esse trabalho tem como objetivo o embasamento teórico e a revisão bibliográfica requerida para a execução do experimento do tubo de impedância acústica, seguindo a norma ISO10534-1(1996), bem como as simulações numéricas. Os dados experimentais são comparados com a simulação numérica do tubo de impedância utilizando o sistema imunológico artificial na caracterização do experimento.

Palavras-chave: Tubo de Impedância Acústica; Monitoramento da Saúde Estrutural; Algoritmo de Seleção Negativa.

ABSTRACT - The structural health monitoring system (SHM), a system that assesses the state of aeronautical, civil or mechanical structures and provides a prediction of their remaining life, has emerged with the need to make structure monitoring more economical and prevent failures. Thus, such a system is defined as a prophylactic, reliable and effective measure against structural failure. This work has objective the theoretical basis and the bibliographic revision for the execution of the acoustic impedance tube experiment following the ISO10534-1 (1996) as well as the numerical simulations. The experimental data are compared with the numerical simulation of the impedance tube using the artificial immune system to characterize the experiment.

Keywords: Acoustic Impedance Tube; Structural Health Monitoring; Negative Selection Algorithm. 


\section{INTRODUÇÃO}

Devido ao desgaste, por fatores temporais, humanos e ambientais, a vida útil de qualquer estrutura, seja aeronáutica, civil ou mecânica, pode ter sua duração encurtada e essa pode apresentar uma falha precoce. Assim, as estruturas necessitam de uma determinada frequência de manutenção para que se evitem falhas.

A indústria com o decorrer da história, e principalmente na contemporaneidade, aumentou os investimentos no desenvolvimento de pesquisas visando a analise e monitoramento da saúde estrutural, evitando catástrofes e danos ambientais, humanitários e econômicos.

Recebe o nome de monitoramento da saúde estrutural (SHM - Structural Health Monitoring) a importante e atual linha de pesquisa que consiste na detecção de falhas em seu estado inicial para que se possa intervir na sua propagação e evitar prejuízos.

Aquisição e processamento de dados, validação e análise de sinais, identificação e caracterização de falhas, interpretação de mudanças adversas em uma estrutura e auxiliar a tomada de decisões são os principais requisitos que um SHM deve atender, como destacado por (HALL, 1999).

A avaliação da integridade de estruturas mecânicas realizada por ensaios não-destrutivos como líquidos penetrantes, partículas magnéticas, ultrassonografia, radiografia, entre outras técnicas tradicionais, apesar de possuir bom aparato instrumental e serem diversamente empregas não satisfazem totalmente as crescentes necessidades industriais (FRANCO et al., 2009).

Estruturas mecânicas como tubos, por exemplo o Gasoduto Bolívia-Brasil, também conhecido como "Gasbol", precisam de constante manutenção e verificação de sua saúde estrutural.

Na seção 3 mostra-se a base teórica para o desenvolvimento e programação de um SHM, na seção 4 os materiais utilizados e a montagem da bancada experimental, na seção 5 o desenvolvimento do projeto. Os resultados da pesquisa são exibidos e discutidos na seção 6 , sendo a seção 7 dedicada as considerações finais.

\section{OBJETIVO DA PESQUISA}

O objetivo desse trabalho de pesquisa é desenvolver um SHMs para estruturas mecânicas utilizando técnicas do algoritmo de seleção negativa (ASN) (FORREST et al., 1994) para realizar a caracterização, analise e monitoramento da integridade estrutural de um tubo de impedância acústica partindo da coleta da pressão acústica em posições no interior do tubo baseando-se na norma ISO10534-1 (1996).

\section{FUNDAMENTAÇÃO TEORICA}

O reconhecimento de padrões exercido pelo sistema imunológico biológico no reconhecimento de linfócitos $T$ que ocorre no timo para categorizar as células como benignas ou malignas é a base da técnica do algoritmo de seleção negativa (ASN) (FORREST et al., 1994). Divide-se o ASN em duas partes: Censoriamento e Monitoramento.

A fase de Censoriamento consiste em determinar um conjunto de vetores próprios protegidos e gerar cadeias aleatórias que constituirão a base-line após avaliar a afinidade entre esses e os sinais próprios de modo a rejeitar determinada cadeia que apresentar afinidade superior ao estipulado. No monitoramento, definida as base-lines, avalia-se a afinidade entre essas e o conjunto de detectores. Caso a afinidade seja superior a um limiar estipulado um elemento nãopróprio é identificado (DE CASTRO, 2001).

As Figuras 1 e 2 ilustram a fase de censoriamento e monitoramento do ASN. 
Figura 1. Fluxograma da fase de censoriamento do ASN.

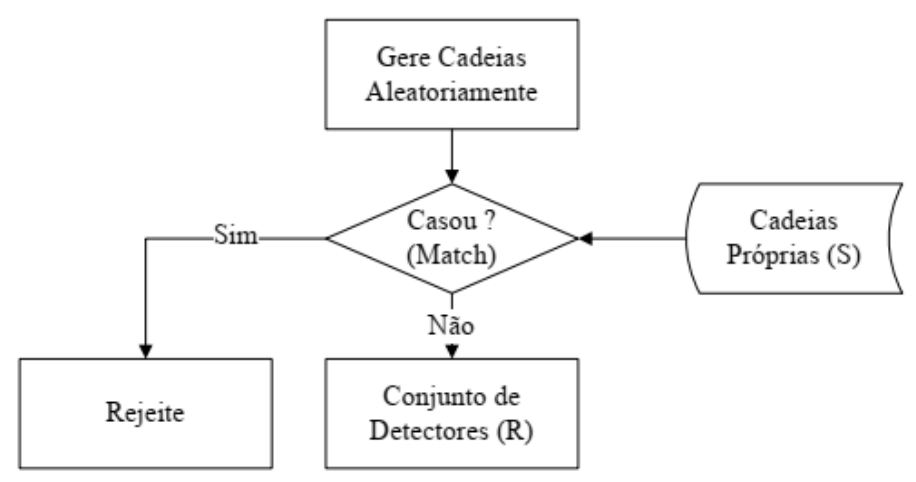

Fonte: (Castro, 2001).

Figura 2. Fluxograma da fase de monitoramento do ASN.

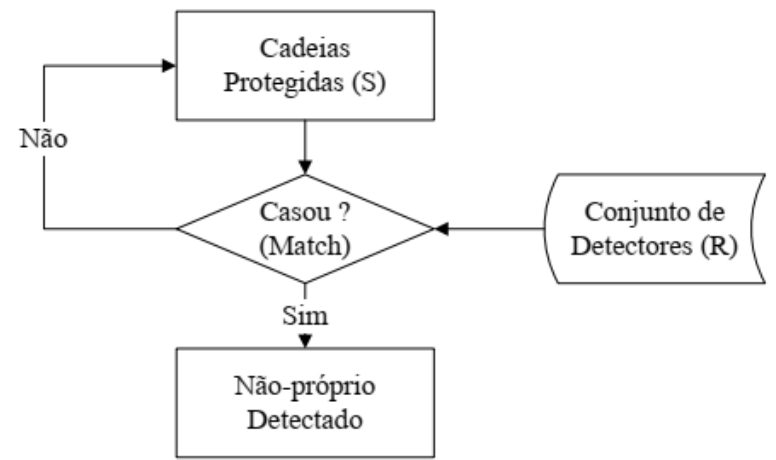

Fonte: (Castro, 2001).

Inicialmente são definidos, na fase de censoriamento, os detectores próprios, cadeias próprias, representando uma situação normal. O conjunto de detectores, com a capacidade de reconhecer padrões não próprios, é gerado em sequência. Cadeias aleatórias são escolhidas, partindo da leitura dos dados. Os detectores funcionam como as células do tipo $T$ maturadas as quais possuem a capacidade de reconhecimento de agentes patogênicos, ou seja, a capacidade de detecção de quase todo elemento não-próprio. A afinidade é verificada comparando cadeias escolhidas de forma aleatória com o conjunto de cadeias próprias. Sendo a afinidade superior a um limiar preestabelecido rejeita-se a cadeia. Senão, coloca-se a cadeia no conjunto de detectores e essa será empregada nas classificações durante o monitoramento dos dados (LIMA, 2013).
A fase seguinte resume-se em monitorar os dados com o intuito de identificar alteração no comportamento das amostras e classificar estas mudanças empregando o conjunto de detectores criados na fase de censoriamento. Então, um elemento não próprio é detectado e classificado quando a afinidade entre as cadeias for superior a um limiar preestabelecido. (LIMA et al., 2016).

O critério conhecido como casamento, que pode ser perfeito ou parcial, é utilizado para avaliar a afinidade entre as cadeias e verificar que são iguais/semelhantes.

Ocorre um casamento perfeito quando todas as posições das cadeias possuem os mesmos valores, isto é, ambas são perfeitamente iguais. Um casamento parcial ocorre quando uma quantidade previamente determinada de posições entre 
as cadeias possui o mesmo valor. Essa quantidade é denominada taxa de afinidade.

O monitoramento da integridade estrutural (Structural Health Monitoring SHM) surge baseado na necessidade industrial de detectar de falhas em seu estado inicial e assim se possa impedir sua propagação e se evitar prejuízos. Um SHM deve atender aos seguintes pontos: Aquisição e processamento de dados, validação e análise de sinais, identificação e caracterização de falhas, interpretação de mudanças adversas em uma estrutura e auxiliar a tomada de decisões (HALL, 1999).

O SHM baseado no algoritmo de seleção negativa possui o diferencial da estabilidade, podendo o sistema aprender com o tempo, e da plasticidade, a capacidade da continua aprendizagem com novos padrões incluídos mantendo o conhecimento anterior (DOEBLING et al., 1998).

Um SHM tendo como base o SIA, em particular o ASN (FORREST et al., 1994), tem seu diagnostico composto por duas fases: Censoriamento e monitoramento. Sendo que, no censoriamento é realizado um censo dos dados para a criação do conjunto de detectores e então o reconhecimento das falhas durante o monitoramento.

Em conformidade com o exibido na Figura 3, a fase de censoriamento é dividida em dois módulos. No modulo de entrada, que compõe a bancada experimental, realizase a aquisição dos sinais. No censoriamento gera-se o conjunto de detectores próprios que será utilizado no monitoramento dos dados. Os detectores próprios são compostos por um grupo de sinais em situação normal, isso é: Sem falhas na estrutura. Eles servem como parâmetro de comparação para identificação de falhas e recebem o nome de base-line. Um sinal coletado da estrutura em uma situação de falha não apresentará afinidade satisfatória com a base-line, e desta forma pode-se identificar a falha presente na estrutura. Já um sinal com a estrutura em situação normal quando comparado com o conjunto de detectores apresentará grande afinidade e não será identificada nenhuma falha (LIMA, 2014). 
Figura 3. Fluxograma da fase de censoriamento do SHM ASN.



Fonte: (Lima, 2014).

O fluxograma da Figura 4 ilustra a fase de monitoramento que também é dividida em duas etapas. Na primeira etapa realiza-se a aquisição dos dados, enquanto que na segunda etapa a discriminação entre os sinais como sendo próprio/não-próprio.

No monitoramento analisa-se os dados em tempo real comparando-os com os detectores criados na fase anterior, buscando facilitar a tomada de decisão através do diagnóstico diferencial.

Feita a aquisição dos sinais executa-se o módulo de deteç̧ão onde compara-se os sinais em analise com os detectores próprios, procurando analisar o casamento entre os sinais.

Sendo a afinidade superior a taxa de afinidade definida os sinais em analise são categorizados como satisfatórios perante o conjunto de detectores e então classificados como sendo da situação normal da estrutura. Caso a afinidade seja menor que a taxa de afinidade o sinal é classificado como uma anormalidade e uma falha estrutural é identificada. 
Figura 4. Fluxograma da fase de monitoramento do SHM ASN.

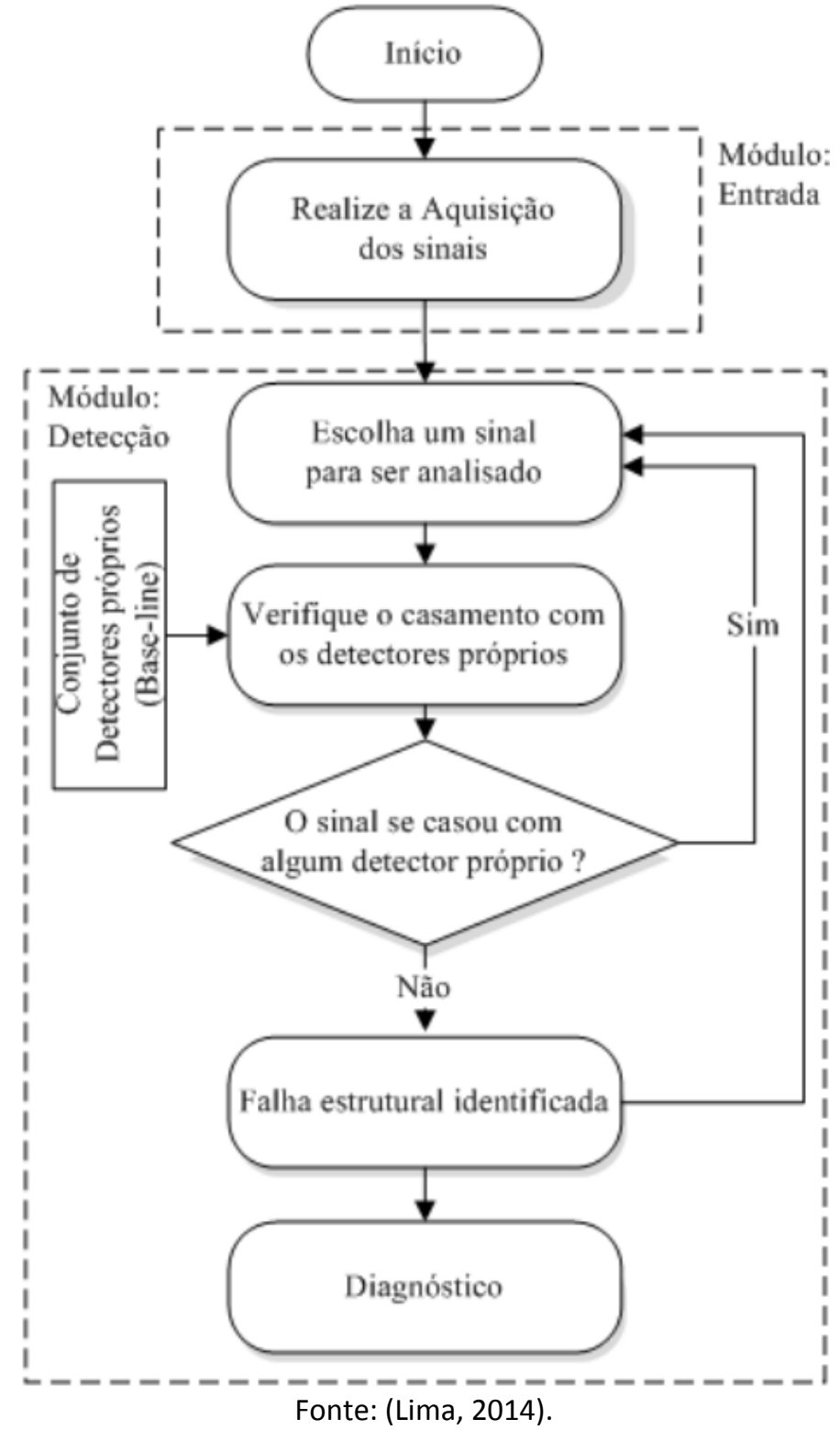

Nesse trabalho utilizasse o critério de casamento parcial proposto por (BRADLEY; TYRRELL, 2002) e um desvio de $30 \%$ nos detectores.

Sendo o objetivo do trabalho a caracterização do experimento a base-line é composta apenas por um sinal normal gerado matematicamente. Os sinais coletados da estrutura, sem falhas, são comparados com esse sinal normal, idealmente o sistema não detectará falhas para que o experimento se dê por caracterizado.

\section{METODOLOGIA ADOTADA}

Esse trabalho baseia-se na norma ISO10534-1(1996). Essa, consiste na utilização de um tubo reto, rígido, de paredes não porosas, sem buracos ou fendas. A secção transversal do tubo utilizado é cilíndrica. A técnica da norma consiste na incidência de uma onda senoidal por uma fonte sonora em uma das extremidades do tubo. Sendo a onda de frequência determinada, propagando no ar. $\mathrm{Na}$ extremidade oposta é firmemente encaixada a tampa com um furo em seu centro. Por essa passa a sonda, com o microfone, que translada axial e internamente ao tubo, coletando as pressões. A Figura 5 esquematiza a montagem do experimento. 
Figura 5. Desenho esquemático da montagem do experimento de bancada.



Fonte: Adaptada de (Outa, 2015, p.3).

Como exibido na Figura 5, o Gerador de Funções é ligado no Amplificador que, por sua vez, é conectado ao alto-falante e ao Osciloscópio. O microfone da sonda, que é conectado a placa de aquisição myDAQ da National Instruments (NI), realiza a coleta do sinal que é armazenado no Notebook, utilizando o National Instruments LabView Student Edition 2013 em conjunto com um algoritmo desenvolvido em Octave para a coleta.

A pressão sonora tanto na ponta interior quanto no exterior da sonda é a mesma. Este fato permite a utilização do microfone na extremidade exterior da sonda que capita a pressão de uma secção do tubo.

A frequência de corte é $2008 \mathrm{~Hz}$, determinada algebricamente em Gerges
(2000, p. 290), para o diâmetro do tubo utilizado $(100 \mathrm{~mm})$; frequências acima deste valor não podem ser estudadas nessas condições.

A amplitude do sinal recebido pela fonte sonora deve respeitar a norma ISO10534-1 (1996), que determina que a onda emitida pela fonte seja preferencialmente $10 \mathrm{~dB}$ maior do que o maior ruído de fundo para a frequência em que se trabalha.

$\mathrm{Na}$ Figura 6 é possível visualizar a bancada experimental montada com os equipamentos utilizados na capitação do sinal. 
Figura 6. Vista Superior da bancada experimental.

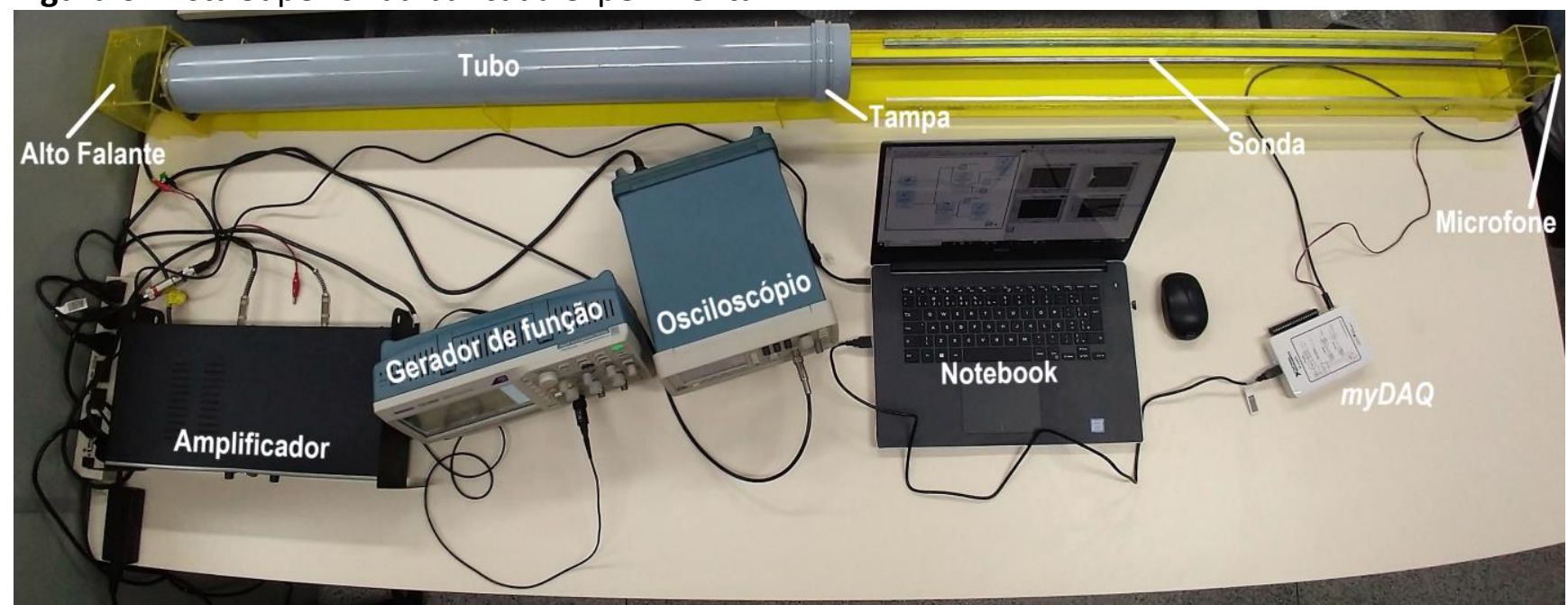

Fonte: Os autores. .

\section{DESENVOLVIMENTO}

Ondas refletidas e transmitidas são geradas quando uma onda acústica plana, que se propaga em um meio I, encontra a superfície limite do seu meio, e início do meio II. Então, a onda refletida é formada no meio I, enquanto a onda transmitida no meio II, como mostra a Figura 7, diz (KINSLER et al., 1999).

Figura 7. Reflexão e transmissão de ondas.

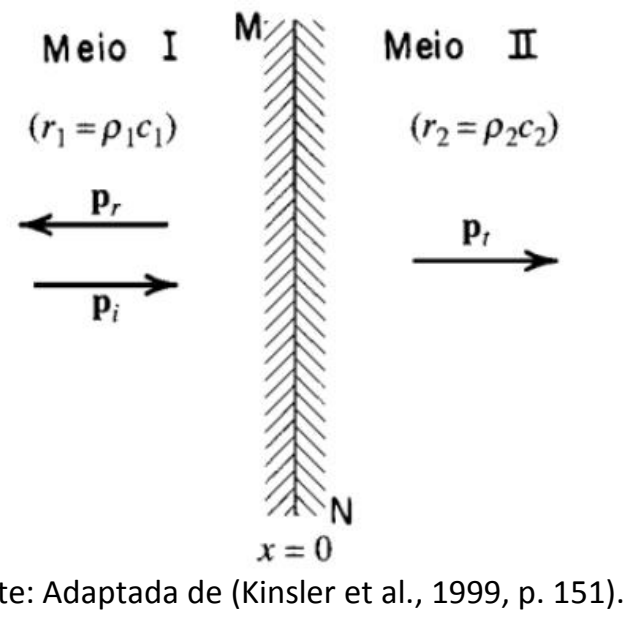

Na Figura 7 a membrana $\mathrm{MN}$ divide os meios I e II. A onda plana progressiva proveniente do Meio I incide normalmente a membrana propagando na direção positiva de $x$, sendo $x$ crescente no sentido do Meio II - a partir do referencial $(x=0)$ adotado na Figura 7.

Com a incidência, a onda refletida forma-se no meio I enquanto a transmitida no meio II (GERGES, 2000).
Internamente ao tubo, com a propagação da onda incidente com uma amplitude adequada, há a formação da onda reincidente, considerando a teoria de transmissão e reflexão em dois meios (KINSLER et al., 1999). Observa-se então o princípio da superposição de ondas no tubo. A somatória da onda incidente com a onda reincidente. A onda obtida dessa superposição recebe o nome de onda estacionaria. 
A sonda translada horizontalmente ao tubo coletando a pressão sonora de cada uma das posições (1 a $100 \mathrm{~cm}$ ), a soma das pressões de cada posição compõe uma coleta. As coletas foram armazenadas em uma matriz com os dados experimentais.
Para a segunda etapa do projeto desenvolveu-se um algoritmo em Octave para a simulação numérica da pressão no interior do tubo. Gerou-se então o sinal normal da Figura 8.

Figura 8. Simulação computacional para frequência de $500 \mathrm{~Hz}$.

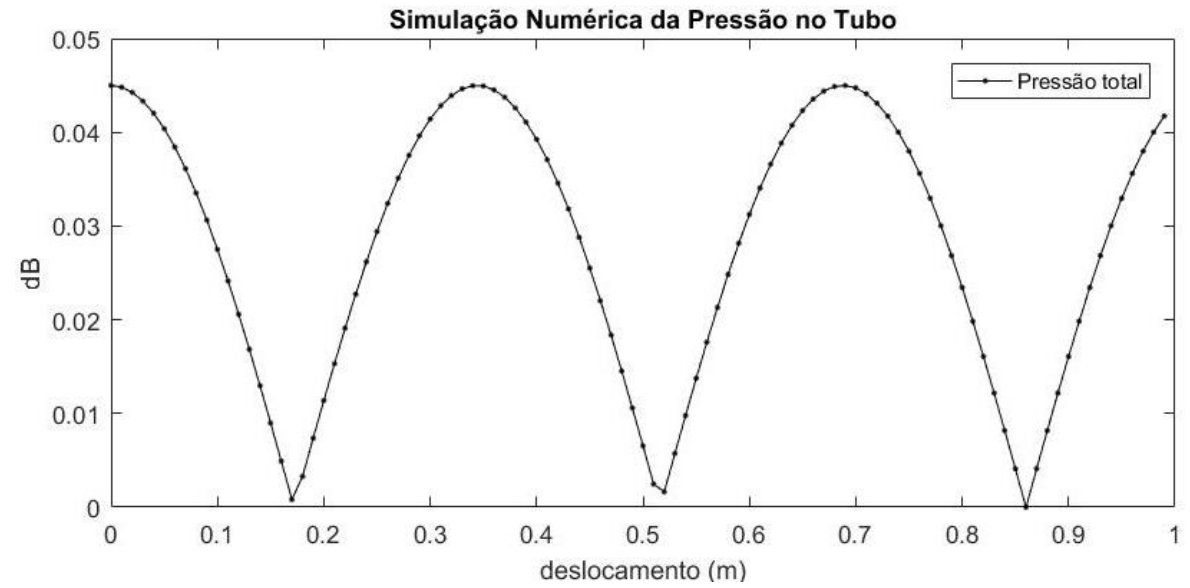

Fonte: Autoria Própria.

\section{RESULTADOS}

Montou-se então um SIA para categorizar a afinidade de cada coleta experimental com o sinal normal simulado computacionalmente via Octave. A Figura 9 exibe graficamente a atuação do SIA com um desvio padrão de $30 \%$ do sinal normal para a frequência de $500 \mathrm{~Hz}$.

Figura 9. Identificação visual do funcionamento do SIA para frequência de $500 \mathrm{~Hz}$.



Fonte: Autoria Própria. 
Na Figura 9, em preto, visualiza-se o sinal normal gerado matematicamente e simulado via Octave. Identifica-se os limites superiores e inferiores do desvio padrão para cada um dos pontos da coleta. As curvas em outras colorações representam as coletas experimentais realizadas no tubo.
A Tabela 1 exibe os resultados obtidos da caracterização experimental realizada pelo SIA, onde encontra-se também a variância e o desvio padrão de cada sinal em relação a curva normal.

Tabela 1. Resultados obtidos do SIA para a caracterização do tubo de impedância para frequência de $500 \mathrm{~Hz}$.

Fonte: Os autores.

\begin{tabular}{|c|c|c|c|}
\hline Coleta & Afinidade & Variância & Desvio Padrão \\
\hline 1 & $77,00 \%$ & 0.0001448 & 0.012033638 \\
\hline 2 & $80,00 \%$ & 0.0001462 & 0.012092891 \\
\hline 3 & $78,00 \%$ & 0.0001479 & 0.012161562 \\
\hline 4 & $75,00 \%$ & 0.0001499 & 0.012244128 \\
\hline 5 & $73,00 \%$ & 0.0001474 & 0.012139549 \\
\hline 6 & $75,00 \%$ & 0.0001487 & 0.012194284 \\
\hline 7 & $77,00 \%$ & 0.0001473 & 0.012135564 \\
\hline 8 & $78,00 \%$ & 0.0001453 & 0.012054074 \\
\hline 9 & $77,00 \%$ & 0.0001451 & 0.012044681 \\
\hline 10 & $75,00 \%$ & 0.0001487 & 0.012192253 \\
\hline
\end{tabular}

Considerando que a taxa de afinidade adotada foi de $70 \%$ a porcentagem de acerto do Sistema Imunológico Artificial foi de $100 \%$ e o experimento foi corretamente caracterizado. O Algoritmo de Seleção Negativa mostrou-se robusto e eficaz na identificação do casamento dos sinais.

\section{CONSIDERAÇÕES FINAIS}

Tratando-se de um experimento vibro-acústico, experimentalmente o sistema apresenta grande sensibilidade. Durante a realização das coletas, pelo trabalho basearse na norma ISO10534-1 (1996), a aquisição dos sinais é demorada, sensível e exaustiva.

Para realizar a aquisição da posição 1 $\mathrm{cm}$ do primeiro sinal experimental (que é composto por cada um dos pontos de 1 a 100 $\mathrm{cm}$ ) deve-se configurar a frequência desejada no gerador de funções, garantir que a amplitude da onda esteja de acordo com a norma ISO10534-1 (1996) regulando, caso necessário, no amplificador. Feito, posicionase a sonda na devida posição e espera-se tempo suficiente para que o sistema entre em regime permanente e ocorra a formação da onda estacionaria interiormente ao tubo. Só então realiza-se a coleta do ponto desejado. Esse procedimento é reproduzido ao menos 10 vezes para cada ponto, para que se tenha a composição dos 10 sinais experimentais.

Satisfatoriamente a caracterização do experimento foi feita com êxito pelo SIA programado, dentro dos parâmetros adotados. A caracterização também foi realizada para outras frequências da banda de oitava. Além da frequência de $500 \mathrm{~Hz}$ apresentada nesse trabalho, se realizou para as frequências de $1000 \mathrm{~Hz}$ e $2000 \mathrm{~Hz}$; ambas abaixo da frequência de corte.

Em continuação a esse trabalho, os próximos passos são a aquisição dos sinais do tubo em situação de falha para então montar-se um SHM para a detecção de falhas estruturais. Pretende-se, ainda, realizar esse procedimento de forma vibracional para então se comparar os métodos. 
AGRADECIMENTOS

Os autores agradecem a Fapesp (Proc.No.2018/16447-8 e Proc.No.2019/ 10515-4), ao Laboratório SISPLEXOS e a UNESP pelo suporte.

\section{REFERÊNCIAS}

DE CASTRO, L. N. Engenharia imunológica: desenvolvimento e aplicação de ferramentas computacionais inspiradas em sistemas imunológicas artificiais. 2001. Tese (Doutorado emEngenharia Elétrica) Faculdade de Engenharia Elétrica e de Computação, Campinas - SP, 2001.

DOEBLING, S. W., FARRAR, C.;PRIME, M. A summary review of vibration-based damage identification methods. The Shock and Vibration Digest, v. 30, p. 91-105. 1998. https://doi.org/10.1177/0583102498030002 $\underline{01}$

FORREST, S. A.; PERELSON, A. L.; CHERUKURI, $R$. Self-nonself discrimination in a computer. In: IEEE SYMPOSIUM ON RESEARCH IN SECURITY AND PRIVACY, 1., 1994, Oakland. Proceedings [...]. Oakland: IEEE, 1994. p. 202212.

FRANCO, V. R.; BUENO, D. D.; BRENNAN, M. J.; CAVALINI JR., A. A.; GONSALEZ, C. G.; LOPES JR., V. Experimental damage location in smart structures using Lamb waves approaches. In: BRAZILIAN CONFERENCE ON DYNAMICS, CONTROL AND THEIR APPLICATIONS - DINCON, 2009. p. 1-4.

GERGES, S. N. Y. Ruído: fundamentos e controles. Florianopolis: NR, 2000. 696 p.

HALL, S. R. The effective management and use of structural health data. In:

INTERNATIONAL WORKSHOP ON

STRUCTURAL HEALTH MONITORING, 1999. p. 265-275.

KINSLER, L. E. et al. Fundamentals of acoustics. 4. ed. Hoboken: John Wiley \& Sons, 1999. $548 \mathrm{p}$.
LIMA, F. P. A. Análise de distúrbios de tensão em sistemas de distribuição de energia elétrica baseada em sistemas imunológicos artificiais. 2013. Dissertação (Mestrado em Engenharia Elétrica) - Faculdade de Engenharia, Universidade Estadual Paulista UNESP, Ilha Solteira - SP, 2013.

LIMA, F. P. A. Monitoramento e identificação de falhas em estruturas aeronáuticas e mecânicas utilizando técnicas de computação inteligente. 2014. Dissertação (Mestrado em Engenharia Mecânica) Faculdade de Engenharia, Universidade Estadual Paulista - UNESP, Ilha Solteira - SP, 2014.

LIMA, F. P. A. Diagnóstico de distúrbios de tensão em sistemas de distribuição baseado num sistema imunológico artificial com aprendizado continuado. 2016. Tese de Doutorado (Engenharia Elétrica) - Faculdade de Engenharia de Ilha Solteira, Ilha Solteira SP, 2016.

OUTRA, R.; CHAVARETTE, F. R. Caracterização do experimento do tubo de impedância de um microfone. 2015. 4 f. Boletim Técnico da FATEC-SP | BT/39 | Jun.,2015. Disponível em: http://bt.fatecsp.br/bulletins/show article/9 90. Acesso em: 2 set 2018. 